\title{
Herschel/SPIRE observations of the dusty disk of NGC $4244^{\star}$
}

\author{
B. W. Holwerda ${ }^{1}$, S. Bianchi ${ }^{2}$, T. Böker ${ }^{1}$, D. Radburn-Smith ${ }^{4}$, R. S. de Jong ${ }^{3}$, M. Baes ${ }^{5}$, P. C. van der Kruit ${ }^{6}$, \\ M. Xilouris ${ }^{7}$, K. D. Gordon ${ }^{8}$, and J. J. Dalcanton ${ }^{4}$ \\ ${ }^{1}$ European Space Agency Research Fellow (ESTEC), Keplerlaan 1, 2200 AG Noordwijk, The Netherlands \\ e-mail: benne.holwerda@esa.int \\ 2 INAF - Arcetri Astrophysical Observatory, Largo Enrico Fermi 5, 50125 Florence, Italy \\ 3 Department of Astronomy, University of Washington, Box 351580, Seattle, WA 98195, USA \\ ${ }^{4}$ Leibniz Institut für Astrophysik Potsdam (AIP), An der Sternwarte 16, 14482 Potsdam, Germany \\ 5 Sterrenkundig Observatorium, Universiteit Gent, Krijgslaan 281-S9, 9000 Gent, Belgium \\ ${ }^{6}$ Kapteyn Astronomical Institute, University of Groningen, PO Box 800, 9700 AV Groningen, The Netherlands \\ 7 Institute of Astronomy and Astrophysics, National Observatory of Athens, P. Penteli, 15236 Athens, Greece \\ ${ }^{8}$ Space Telescope Science Institute, 3700 San Martin Drive, MD 21218, Baltimore, USA \\ Received 9 December 2011 / Accepted 19 March 2012
}

\section{ABSTRACT}

\begin{abstract}
We present Herschel/SPIRE images at 250, 350, and $500 \mu \mathrm{m}$ of NGC 4244, a typical low-mass, disk-only and edge-on spiral galaxy. The dust disk is clumpy and shows signs of truncation at the break radius of the stellar disk. This disk coincides with the densest part of the Hi disk. We compare the spectral energy distribution (SED), including the new SPIRE fluxes, to 3D radiative transfer models; a smooth model disk and a clumpy model with embedded heating. Each model requires a very high value for the dust scale-length $\left(h_{\mathrm{d}}=2-5 h_{*}\right)$, higher dust masses than previous models of NGC $4244\left(M_{\mathrm{d}}=0.47-1.39 \times 10^{7} M_{\odot}\right)$ and a face-on optical depth of $\tau_{V}^{\text {f.o. }}=0.4-1.12$, in agreement with previous disk opacity studies. The vertical scales of stars and dust are similar. The clumpy model much better mimics the general morphology in the sub-mm images and the general SED. The inferred gas-to-dust mass ratio is compatible with those of similar low-mass disks. The relatively large radial scale-length of the dust disk points to radial mixing of the dusty ISM within the stellar disk. The large vertical dust scale and the clumpy dust distribution of our SED model are both consistent with a scenario in which the vertical structure of the ISM is dictated by the balance of turbulence and self-gravity.
\end{abstract}

Key words. radiative transfer - dust, extinction - ISM: structure - galaxies: ISM - galaxies: spiral - galaxies: structure

\section{Introduction}

The edge-on perspective of a spiral galaxy's disk reveals both the vertical structure of the disk and its faint outskirts, thanks to line-of-sight integration. One typical feature is the mid-plane dust absorption lane. Dust is linked to the cold molecular ISM, mechanically (Allen et al. 1986; Weingartner \& Draine 2001), through mutual shielding, and as a catalyst for molecular gas formation. Dalcanton et al. (2004) used the appearance of dust lanes as a probe of vertical stability of spiral disks. They found that in massive spirals, the ISM collapses into a thin dust-lane, while in low-mass disks $\left(v_{\text {rot }}<120 \mathrm{~km} \mathrm{~s}^{-1}\right)$ the dust morphology is flocculant.

One comprehensive approach to investigate this phenomenon is to model the multi-wavelength information on a range of spiral disks with a spectral energy distribution (SED) model. Dust absorption, especially within the thin dark dust lane, is strongest at optical and ultra-violet wavelengths and the challenge for the models is to balance the observed absorption with the dust emission at far-infrared (FIR) and sub-millimeter (mm) wavelengths (e.g., Popescu et al. 2000; Misselt et al. 2001; Gordon et al. 2001; Alton et al. 2004; Bianchi 2008; Baes et al. 2010; Bianchi \& Xilouris 2011). Until recently, these models were degenerate in the vertical distributions of stellar light and

\footnotetext{
* Herschel is an ESA space observatory with science instruments provided by European-led Principal Investigator consortia and with important participation from NASA.
}

ISM due to a lack of resolution and wavelength coverage in the FIR and sub-mm, but with the advent of the Herschel Space Observatory, the vertical structure of nearby edge-on disks can now be fully resolved.

Massive edge-on spiral galaxies have been observed by several Herschel surveys (see Boselli et al. 2010; Eales et al. 2010b; Davies et al. 2010) and a dedicated program ${ }^{1}$ (Verstappen et al. in prep.) which targets seven massive spirals. Our NHEMESES ${ }^{2}$ program aims to observe a complementary sample of low-mass nearby edge-ons. The combined observations will serve as a test for the different radiative transfer models in the literature; those from Baes et al. (2003, 2011), Bianchi (2007, 2008), Gordon et al. (2001), Misselt et al. (2001) and Pierini et al. (2004), and Popescu et al. (2000, 2011). These models will further test the suggested dichotomy in the vertical dust structure.

Here, we explore the first NHEMESES results on NGC 4244, the prototypical low-mass $\left(v_{\text {rot }}=95 \mathrm{~km} \mathrm{~s}^{-1}\right)$, latetype (Sc), edge-on spiral at a distance ${ }^{3}$ of $4.4 \mathrm{Mpc}$ to compare to the first Herschel results on the massive edge-on spiral NGC 891 (Bianchi \& Xilouris 2011). Originally, van der Kruit \& Searle (1981) found a single edge-on exponential disk truncated at $9 \mathrm{kpc}$, later confirmed by Fry et al. (1999). The truncation

\footnotetext{
${ }^{1}$ HERschel Observations of Edge-on Spirals, HEROES.

2 New HErschel Multi-wavelength Extragalactic Survey of Edge-on Spirals (Holwerda et al. 2011).

3 The mean of the distances in NED from Heald et al. (2011b). Radburn-Smith et al. (2011) derive a distance of $4.7 \mathrm{Mpc}$.
} 


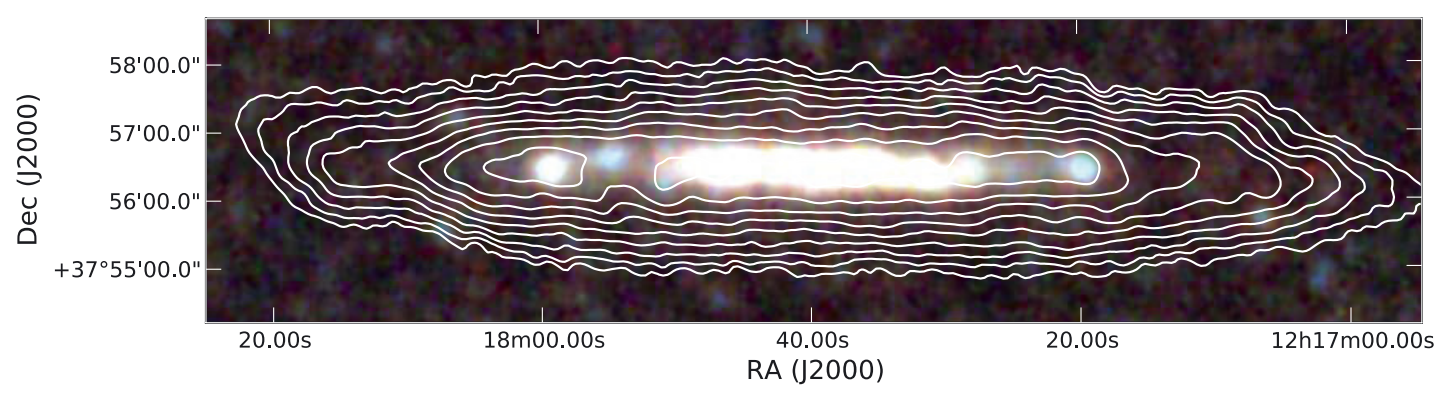

Fig. 1. A color composite of the SPIRE maps of NGC 4244, 250 (red), 350 (green), and $500 \mu \mathrm{m}$ (blue) with the $\mathrm{HI}$ contours from Zschaechner et al. (2011) (contours at $6.4 \times 10^{19} \mathrm{~cm}^{-2}$ increasing by factors of 2 . The SPIRE flux stays within the $8.1 \times 10^{19} \mathrm{~cm}^{-2}$ contour (second to highest).

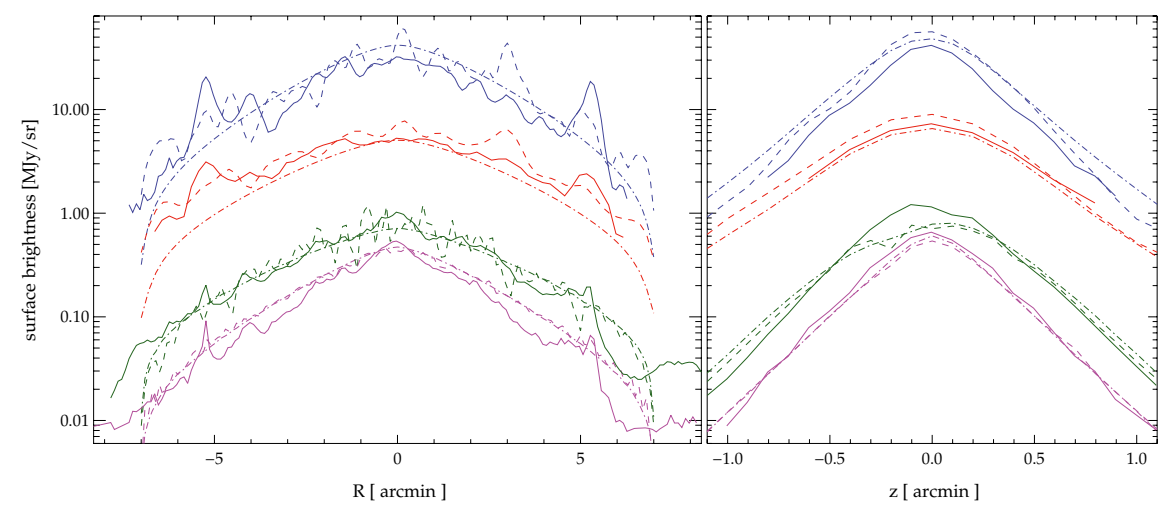

Fig. 2. Major (left) and minor (right) axis profiles, averaged over a strip of $30^{\prime \prime}$ (solid lines), in the sDss-R (green) and IRAC $3.6 \mu \mathrm{m}$ (pink, offset by a factor 2 for clarity) and the 250 (blue) and 500 (red) $\mu \mathrm{m}$ SPIRE data, all at native resolution. The major axis profile spans $D_{25}=16.6$ (RC3). We also show the smooth (dot-dash) and clumpy (dashed) TRADING models for comparison (convolved with the SPIRE PSFs at 250 and $500 \mu \mathrm{m}$ ). We assumed $\mathrm{PA}=47.3^{\circ}$ (de Jong et al. 2007). was also observed with the Hubble Space Telescope (de Jong et al. 2007). Yoachim \& Dalcanton (2006) note a second, thick stellar disk as doubtful, but Comerón et al. (2011) report both a thin and thick disk of equal mass. Its center hosts a rotating nuclear star-cluster (Seth et al. 2008), and the disk is classified as "corrugated" (Florido et al. 1991).

NGC 4244's ISM is mostly in a thin disk, both the ionized gas (Hoopes et al. 1999) and warm dust, implying a low specific star-formation rate (Kodaira \& Yamashita 1996). The Hi disk shows a clear warp, kinematic evidence for a compact dark matter halo, and lagging extra-planar gas (Olling 1996; Zschaechner et al. 2011; Heald et al. 2011a). The $H_{2}$ mass, based on CO observations, is $1.4 \times 10^{7} M_{\odot}$ (Matthews \& Wood 2001).

A recent SED model by MacLachlan et al. (2011) finds that most $(80 \%)$ of the FIR emission could arise from an optically thin disk with a similar scale-height for both dust and stars (as did Seth et al. 2005, based on stellar populations), a dust scalelength 1.8 times the stellar one and a dust mass of $2.38 \times 10^{6} M_{\odot}$. They note their model diverges from the observed disk in the FIR and that the dust model is still uncertain without sub-mm observations.

In this Letter, we present new SPIRE sub-mm observations of NGC 4244, adding spatial information on the cold dust, and present two TRADING models to illustrate the role of ISM geometry on the observed overall SED and the sub-mm images.

\section{Observations}

SPIRE (Spectral and Photometric Imaging REceiver, Griffin et al. 2010) instrument at 250, 350 and $500 \mu \mathrm{m}$ onboard the ESA Herschel Space Observatory (Pilbratt et al. 2010) observed NGC 4244 at 250, 350 and $500 \mu \mathrm{m}$ in large map mode, over $25^{\prime} \times 25^{\prime}$ centered on the object with two cross-scans, using a $30^{\prime \prime} \mathrm{s}^{-1}$ scan rate. Data-reduction is as in Bianchi \& Xilouris (2011); we generated maps with the naïve map-making procedure within HIPE (Ott 2010), with pixel sizes of 6", 8", $12^{\prime \prime}$ at 250,350 and $500 \mu \mathrm{m}$, respectively (about $1 / 3$ of the measured FWHM: $\approx 18^{\prime \prime}, 25^{\prime \prime}$ and $36^{\prime \prime}$ ), resulting in uniform backgrounds and sky rms noise of $1,0.6$ and $0.3 \mathrm{MJy} \mathrm{sr}^{-1}$ respectively, Fig. 1). A color correction half-way between that for a pointand extended-source was implemented with a color-correction in between the point- and extended-source (marginally resolved vertically), we derive integrated fluxes of $15.9,9.8$, and $5.1 \mathrm{Jy}$ $(250,350$ and $500 \mu \mathrm{m})$, and adopt a conservative $15 \%$ calibration error.

\section{Results and analysis}

Figure 1 shows a SPIRE color-composite, together with the Hi contours from Zschaechner et al. (2011). The dust emission falls entirely within the second highest Hi contour, concentrated in a flat, single disk with no emission in the extended warped Hi disk and envelope, in contrast to the more massive NGC 891, where Popescu \& Tuffs (2003) and Bianchi \& Xilouris (2011) find evidence for dust throughout the Hi disk and the extended envelope (Oosterloo et al. 2007). Dust is concentrated in the thin Hi disk, but we note that dust and Hi clumps do not coincide.

Figure 2 shows the radial and vertical profiles of the sub-mm data, SDSs-R data (Aihara et al. 2011), and the Spitzer $3.6 \mu \mathrm{m}$ image from a related Spitzer program ${ }^{4}$. The sub-mm flux is contained within the $D_{25}$ radius, and the profile shows clumps of emission at both ends of the disk. These clumps of sub-mm emission are the last reliable detection of the disk before the truncation radius (420", $9 \mathrm{kpc}$ van der Kruit \& Searle 1981). The vertical sub-mm profiles are similar in width to those in Spitzer 3.6 $\mu \mathrm{m}$ emission, a good tracer of the stellar mass (e.g. Meidt et al. 2012). Figure 2 suggest that dust is confined to the thin disk, in contrast to NGC 891, where a second vertical component is observed (Kamphuis et al. 2007; Bianchi \& Xilouris 2011), as well as a thin dust lane (Xilouris et al. 1999; Schechtman-Rook et al. 2012a,b).

Figure 3 shows the optical and infrared SED of the galaxy. The SPIRE flux densities show a spectral index $F_{v} \propto v^{1.6}$, flatter

${ }^{4}$ P.I. R. S. de Jong, Holwerda et al., in prep. 


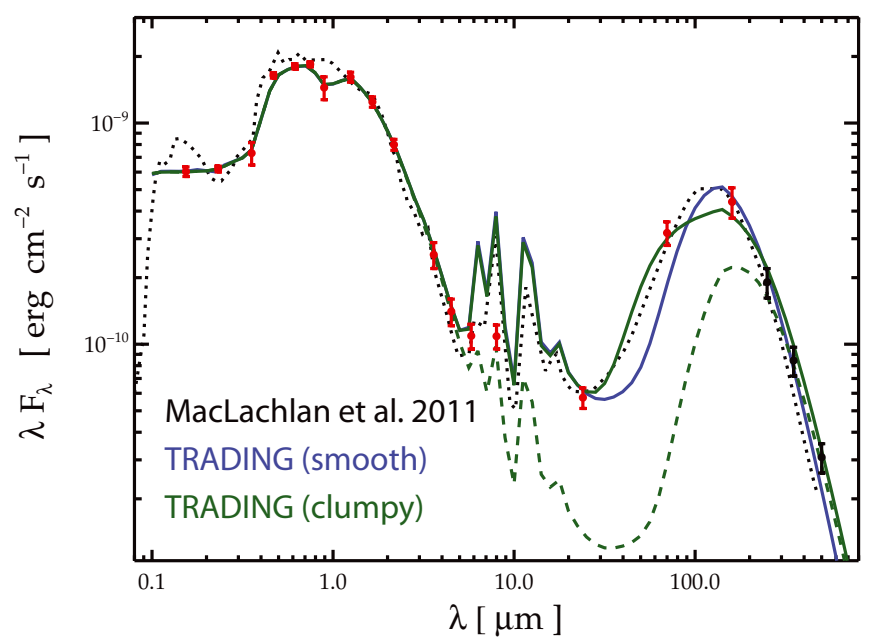

Fig. 3. The spectral energy distribution of NGC 4244. The Herschel/SPIRE fluxes from this work (black points) are show together with literature data (red symbols, Dale et al. 2009; MacLachlan et al. 2011). We show the smooth model (blue line); the clumpy model with and without embedded stellar emission (solid and dashed green line, respectively), and the model from MacLachlan et al. (black dotted line).

than the more massive NGC $891\left(F_{v} \propto v^{2.7}\right)$, and thus indicative of a colder dust temperature (or of different dust properties). When compared to the radiative transfer model of MacLachlan et al. (2011), the observed fluxes at 350 and $500 \mu \mathrm{m}$ are higher by a factor two (Fig. 3, Holwerda et al. 2011).

We model the surface brightness distribution and SED of NGC 4244 using the radiative transfer code TRADING (Bianchi 2008). We initialize the stellar distribution with the scale-length and height $\left(h_{*}, z_{*}\right)$ as derived by Fry et al. (1999) (scaled to 4.4 Mpc), and adopted an intrinsic SED that, after being processed by dust, reproduces the observed stellar fluxes in the optical and NIR. We truncate the model, for both stars and dust, at $420^{\prime \prime}(9 \mathrm{kpc})$ since it is to this radius we have reliable fluxes for both.

First, we try a smooth exponential dust disk with the same scale-height $\left(z_{\mathrm{d}}\right)$ as for stars, and modify the scale-length $\left(h_{\mathrm{d}}\right)$ and the central $V$-band face-on optical depth though the whole disk $\left(\tau_{V}^{\text {f.o. }}\right)$ to approximate both the sub-mm surface brightness profiles (Fig. 2) and the SED (Fig. 3). As MacLachlan et al. (2011), we require a dust scale-length larger than the stellar one to match the observed major axis profiles. However, contrary to MacLachlan et al. (2011), we need a higher opacity (and hence dust mass) to match the peak of the SED $^{5}$. Ours and MacLachlan et al. (2011) models are compared in Table 1. While the smooth model can broadly match the peak of the SED and the profiles, it lacks hot dust to explain emission at $70 \mu \mathrm{m}$. And it underpredicts the $500 \mu \mathrm{m}$ flux, implying a lower dust temperature ${ }^{6}$.

Secondly, we consider an exclusively clumpy disk model; an exponential distribution of Giant Molecular Clouds (GMC), similar to the clumpy part of the smooth+clumpy model in Bianchi (2008) with $M_{\mathrm{GMC}}=10^{6.5} M_{\odot}$, gas-to-dust mass ratio $=100$, $R_{\mathrm{GMC}} \simeq 100$ pc. Bianchi (2008) find the SED does not depend

\footnotetext{
5 Although the model of MacLachlan et al. (2011) includes a distribution of compact sources, with a dust mass about ten times than that of their diffuse disk, its contribution to the peak SED, as estimated from their Fig. 12, is smaller than $15 \%$.

${ }^{6}$ Our assumption is that the dust grain population of NGC 4244 and Milky Way are identical but differences in sub-mm flux and optical extinction can be due to different grain distributions as well.
}

Table 1. Results from the SED models.

\begin{tabular}{lcccc}
\hline \hline Parameter & $\begin{array}{c}\text { MacLachlan } \\
\text { et al. }(2011)\end{array}$ & $\begin{array}{c}\text { Trading } \\
\text { Smooth }\end{array}$ & Clumped & Unit \\
\hline$h_{*}$ & 1.9 & 2.3 & 2.3 & $\mathrm{kpc}$ \\
$z_{*}, z_{\mathrm{d}}$ & 0.2 & 0.3 & 0.3 & $\mathrm{kpc}$ \\
$h_{\mathrm{d}}$ & 3.4 & $4.5\left(2 h_{*}\right)$ & $9=r_{\text {trunc }}$ & $\mathrm{kpc}$ \\
$r_{\text {trunc }}$ & - & 9 & 9 & $\mathrm{kpc}$ \\
$L_{*}$ & $3.4 \times 10^{9}$ & $3.9 \times 10^{9}$ & $3.0 \times 10^{9}$ & $L_{\odot}$ \\
$M_{\mathrm{d}}$ & $2.4 \times 10^{6}$ & $0.5 \times 10^{7}$ & $1.4 \times 10^{7}$ & $M_{\odot}$ \\
$i$ & $84.5^{\circ}$ & $88^{\circ}$ & $88^{\circ}$ & \\
$L_{\text {cloud }}$ & $1.0 \times 10^{8}$ & - & $3.7 \times 10^{8}$ & $L_{\odot}$ \\
$\tau_{V}^{\text {fo. }}$ & 0.2 & 0.4 & 1.1 & \\
$h_{*} / z_{*}$ & 9.5 & 7.5 & 7.5 & $8.0(3.6 \mu \mathrm{m})$ \\
$h_{\mathrm{d}} / z_{\mathrm{d}}$ & 17 & 15 & 30 & $10(8.0 \mu \mathrm{m})$ \\
\hline
\end{tabular}

critically on the GMC size distribution. This model matches the sub-mm SED well but fails in the FIR (Fig. 3, dashed line). To match the $70 \mu \mathrm{m}$ emission, we heat $60 \%$ of the clouds internally with a hot young starcluster $\left(T=35000 \mathrm{~K}, 1.56 \times 10^{6} L_{\odot}\right.$, Fig. 3 solid green line), similar to the second dense dust disk with young stars introduced by Popescu et al. (2000) to reconcile FIR and sub-mm fluxes. We note that PAH fluxes (e.g., Spitzer $8 \mu \mathrm{m}$ ) are not yet reproduced by this model but this may be solved with a range of cloud sizes and heating source types in a future iteration. The resulting heated clumpy model requires a large dust scale-length, $h_{\mathrm{d}} \sim r_{\text {trunc }}$, an increase in total dust mass (three times the smooth model mass) and some $60 \%$ of the GMCs to be internally heated (equivalent to $\sim 12 \% L_{\text {bol }}$ of the stellar disk).

The smooth and partially heated clumpy SED models illustrate how, in the case of a small disk as NGC 4244, highresolution sub-mm data reveals a dust disk with more cold dust than a FIR-only model, which is distributed more in clumps and throughout the stellar disk (similar scale-length and height as the stellar disk). A complete fit of the SED (including future Herschel/PACS observations) will be presented in the context of our complete low-mass edge-on sample.

\section{Discussion}

Stacked observations in the Herschel H-ATLAS survey (Eales et al. 2010a) point to specific characteristics $\left(T_{\mathrm{d}} \sim 15 \mathrm{~K}\right.$, $\left.M_{\mathrm{d}} \sim 10^{7} M_{\odot}\right)$ for blue, low-mass spirals $\left(\sim 10^{9} M_{\odot}\right.$ stellar mass Bourne et al. 2012). NGC 4244's stellar and Hi mass are 6 and $2.5 \times 10^{9} M_{\odot}$ (Strickland et al. 2004; Zschaechner et al. 2011). Compared to the H-ATLAS results, our SED dust mass is reasonable and the gas-to-dust ratio (535-170 for the smooth and clumped model respectively) is in line with those found for smaller spiral disks (Leroy et al. 2009).

The central optical depth of $\tau_{V}^{\text {f.o. }} \sim 0.45-1.12$ is similar to the face-on optical depths in occulting galaxies (White et al. 2000; Holwerda et al. 2007b) or distant galaxy counts (Holwerda et al. 2005, 2007a,c). We therefore feel confident that our SED model accurately reflects the true dust mass of NGC 4244.

The scale-length of the dust in the SED models is consistently much larger to the stellar one; a factor 1.8 to 4.9 , depending on the model. For NGC 891, a ratio of $1.5-2$ is typically found (Xilouris et al. 1999; Popescu et al. 2000, 2011; Driver et al. 2007; Bianchi 2008; Bianchi \& Xilouris 2011). Similarly, Holwerda et al. (2009) and Holwerda et al. (2012) report a clumpy, extended and flat dust profile in a similar mass face-on galaxy occulting a distant bright bulge (2MASX J00482185-2507365, near NGC 253). These flat radial profiles of dust suggest radial mixing of the ISM to a similar 
level found in the stellar disks (de Jong et al. 2007; Roškar et al. 2008, Radburn-Smith et al. 2012).

We find a dust scale-height similar to the stellar one, as do MacLachlan et al. (2011). They attribute this to either the flattening of dust disks (Dalcanton et al. 2004) or a smaller stellar scale-height due to inefficient dynamical heating of the stellar disk in lower mass galaxies (Bizyaev \& Kajsin 2004). To distinguish between these two scenarios, we compare the oblateness $(h / z)$ of the stellar and dust disk (Table 1) to those in Fig. 2 in Dalcanton et al. (2004). We also include the oblateness of the 3.6 and $8.0 \mu \mathrm{m}$ Spitzer observations (Holwerda et al., in prep.). We note that the value for $z_{*}$ of $300 \mathrm{pc}$ is the scale-height of the thin disk found by Comerón et al. (2011) and RGB stars (Seth et al. 2005). NGC 4244's stellar disk oblateness is at the highest, i.e., flattest value for low-mass disks, similar to the oblateness of massive disks, arguing against the less efficient disk heating compared to massive disks. The dusty disk oblateness is extremely high, even compared to the PAH emission $(h / z=10$ at $8 \mu \mathrm{m})$, but this is predominantly because of the large scalelength of the dust disk. The similar stellar and dust scale-height and the best match by clumpy SED model are both consistent with the scenario from Dalcanton et al. (2004); in a low-mass disk such as this one, the ISM does not collapse into a thin plane but remains fractured and spread throughout the height of the stellar disk.

\section{Summary and conclusions}

Based on new sub-mm observations with the Herschel Space Observatory of NGC 4244, and some illustrative models with the TRADING code, we find that:

i. The cold dust is confined to the inner, flat Hi disk (Fig. 1).

ii. The dusty ISM is confined to the stellar radius (Fig. 2).

iii. A smooth model under-predicts 70 and $500 \mu \mathrm{m}$ fluxes (Fig. 3).

iv. A clumpy dust disk model with a fraction of the clumps heated by embedded sources fits both the general SED (Fig. 3) and the morphology of the disk (Fig. 2) in sub-mm well.

vi. The inferred scales of stars and dust are consistent with a scenario that includes some radial migration of dust and a vertical ISM scale supported by turbulence (Table 1).

Future improvements of the NGC 4244 SED model includes new FIR (Herschel/PACS) information to constrain the cold dust structure and the number of GMCs and the stellar type of their heating sources. We plan to put these results in the context of the full NHEMESES and HEROES Herschel observations to infer if there is indeed a phase-change with mass in the dust structure and additionally to benchmark current SED models.

The authors thank ESA for the Fellowship Program and operation of the Herschel Space Observatory, John MacLachlan for his SED data and Laura Zschaechner for her Hi map and the anonymous referee for his or her diligent work.

\section{References}

Aihara, H., Allende Prieto, C., An, D., et al. 2011, ApJS, 193, 29

Allen, R. J., Atherton, P. D., \& Tilanus, R. P. J. 1986, Nature, 319, 296

Alton, P. B., Xilouris, E. M., Misiriotis, A., Dasyra, K. M., \& Dumke, M. 2004, A\&A, 425, 109
Baes, M., Davies, J. I., Dejonghe, H., et al. 2003, MNRAS, 343, 1081

Baes, M., Fritz, J., Gadotti, D. A., et al. 2010, A\&A, 518, L39

Baes, M., Verstappen, J., De Looze, I., et al. 2011, ApJS, 196, 22

Bianchi, S. 2007, A\&A, 471, 765

Bianchi, S. 2008, A\&A, 490, 461

Bianchi, S., \& Xilouris, E. M. 2011, A\&A, 531, L11

Bizyaev, D., \& Kajsin, S. 2004, ApJ, 613, 886

Boselli, A., Eales, S., Cortese, L., et al. 2010, PASP, 122, 261

Bourne, N., Maddox, S. J., Dunne, L., et al. 2012, MNRAS, 421, 3027

Comerón, S., Knapen, J. H., Sheth, K., et al. 2011, ApJ, 729, 18

Dalcanton, J. J., Yoachim, P., \& Bernstein, R. A. 2004, ApJ, 608, 189

Dale, D. A., Cohen, S. A., Johnson, L. C., et al. 2009, ApJ, 703, 517

Davies, J. I., Baes, M., Bendo, G. J., et al. 2010, A\&A, 518, L48

de Jong, R. S., Seth, A. C., Radburn-Smith, D. J., et al. 2007, ApJ, 667, L49

Driver, S. P., Popescu, C. C., Tuffs, R. J., et al. 2007, MNRAS, 563

Eales, S., Dunne, L., Clements, D., et al. 2010a, PASP, 122, 499

Eales, S. A., Smith, M. W. L., Wilson, C. D., et al. 2010b, A\&A, 518, L62

Florido, E., Battaner, E., Sanchez-Saavedra, M. L., Prieto, M., \& Mediavilla, E. 1991, MNRAS, 251, 193

Fry, A. M., Morrison, H. L., Harding, P., \& Boroson, T. A. 1999, AJ, 118, 1209

Gordon, K. D., Misselt, K. A., Witt, A. N., \& Clayton, G. C. 2001, ApJ, 551, 269

Griffin, M. J., Abergel, A., Abreu, A., et al. 2010, A\&A, 518, L3

Heald, G., Allan, J., Zschaechner, L., et al. 2011a, IAU Symp. 277, in ed. C. Carignan, F. Combes, \& K. C. Freeman, 59

Heald, G., Józsa, G., Serra, P., et al. 2011b, A\&A, 526, A118

Holwerda, B. W., González, R. A., Allen, R. J., \& van der Kruit, P. C. 2005, AJ, 129,1396

Holwerda, B. W., Draine, B., Gordon, K. D., et al. 2007a, AJ, 134, 2226

Holwerda, B. W., Keel, W. C., \& Bolton, A. 2007b, AJ, 134, 2385

Holwerda, B. W., Meyer, M., Regan, M., et al. 2007c, AJ, 134, 1655

Holwerda, B. W., Keel, W. C., Williams, B., Dalcanton, J. J., \& de Jong, R. S. 2009, AJ, 137, 3000

Holwerda, B. W., Bianchi, S., Baes, M., et al. 2011 [arXiv: 1109. 5603]

Holwerda, B. W., Dalcanton, J. J., Keel, W. C., Boeker, T., \& de Jong, R. S. 2012, MNRAS, submitted

Hoopes, C. G., Walterbos, R. A. M., \& Rand, R. J. 1999, ApJ, 522, 669

Kamphuis, P., Holwerda, B. W., Allen, R. J., Peletier, R. F., \& van der Kruit, P. C. 2007, A\&A, 471, L1

Kodaira, K., \& Yamashita, T. 1996, PASJ, 48, 581

Leroy, A. K., Walter, F., Bigiel, F., et al. 2009, AJ, 137, 4670

MacLachlan, J. M., Matthews, L. D., Wood, K., \& Gallagher, J. S. 2011, ApJ, 741,6

Matthews, L. D., \& Wood, K. 2001, ApJ, 548, 150

Meidt, S. E., Schinnerer, E., Knapen, J. H., et al. 2012, ApJ, 744, 17

Misselt, K. A., Gordon, K. D., Clayton, G. C., \& Wolff, M. J. 2001, ApJ, 551, 277

Olling, R. P. 1996, AJ, 112, 457

Oosterloo, T., Fraternali, F., \& Sancisi, R. 2007, AJ, 134, 1019

Ott, S. 2010, in Astronomical Data Analysis Software and Systems XIX, ed. Y. Mizumoto, K.-I. Morita, \& M. Ohishi, ASP Conf. Ser., 434, 139

Pierini, D., Gordon, K. D., Witt, A. N., \& Madsen, G. J. 2004, ApJ, 617, 1022

Pilbratt, G. L., Riedinger, J. R., Passvogel, T., et al. 2010, A\&A, 518, L1

Popescu, C. C., \& Tuffs, R. J. 2003, A\&A, 410, L21

Popescu, C. C., Misiriotis, A., Kylafis, N. D., Tuffs, R. J., \& Fischera, J. 2000, A\&A, 362, 138

Popescu, C. C., Tuffs, R. J., Dopita, M. A., et al. 2011, A\&A, 527, A109

Radburn-Smith, D. J., de Jong, R. S., Seth, A. C., et al. 2011, ApJS, 195, 18

Radburn-Smith, D. J., Röskar, R., Dalcanton, J. J., et al. 2012, ApJ, accepted

Roškar, R., Debattista, V. P., Stinson, G. S., et al. 2008, ApJ, 675, L65

Schechtman-Rook, A., Bershady, M. A., \& Wood, K. 2012a, ApJ, 746, 70

Schechtman-Rook, A., Bershady, M. A., Wood, K., \& Robitaille, T. P. 2012b [arXiv: 1203.0023]

Seth, A. C., Dalcanton, J. J., \& de Jong, R. S. 2005, AJ, 129, 1331

Seth, A., Agüeros, M., Lee, D., \& Basu-Zych, A. 2008, ApJ, 678, 116

Strickland, D. K., Heckman, T. M., Colbert, E. J. M., Hoopes, C. G., \& Weaver, K. A. 2004, ApJS, 151, 193

van der Kruit, P. C., \& Searle, L. 1981, A\&A, 95, 105

Weingartner, J. C., \& Draine, B. T. 2001, ApJ, 553, 581

White, III, R. E., Keel, W. C., \& Conselice, C. J. 2000, ApJ, 542, 761

Xilouris, E. M., Byun, Y. I., Kylafis, N. D., Paleologou, E. V., \& Papamastorakis, J. 1999, A\&A, 344, 868

Yoachim, P., \& Dalcanton, J. J. 2006, AJ, 131, 226

Zschaechner, L. K., Rand, R. J., Heald, G. H., Gentile, G., \& Kamphuis, P. 2011, ApJ, 740, 35 